\title{
140.
}

\section{RESEARCHES ON THE PARTITION OF NUMBERS.}

[From the Philosophical Transactions of the Royal Society of London, vol. CxLV for the year 1855, pp. 127-140. Received April 14,-Read May 24, 1855.]

I PROPOSE to discuss the following problem: "To find in how many ways a number $q$ can be made up of the elements $a, b, c, \ldots$ each element being repeatable an indefinite number of times." The required number of partitions is represented by the notation

$$
P(a, b, c, \ldots) q
$$

and we have, as is well known,

$$
P(a, b, c, \ldots) q=\text { coefficient } x^{q} \text { in } \frac{1}{\left(1-x^{a}\right)\left(1-x^{b}\right)\left(1-x^{c}\right) \ldots},
$$

where the expansion is to be effected in ascending powers of $x$.

It may be as well to remark that each element is to be considered as a separate and distinct element, notwithstanding any equalities which may exist between the numbers $a, b, c, \ldots$; thus, although $a=b$, yet $a+a+a+\& c$. and $a+a+b+\&$ c. are to be considered as two different partitions of the number $q$, and so in all similar cases.

The solution of the problem is thus seen to depend upon the theory, to which I now proceed, of the expansion of algebraical fractions.

Consider an algebraical fraction $\frac{\phi x}{f x}$,

where the denominator is the product of any number of factors (the same or different) of the form $1-x^{m}$. Suppose in general that $\left[1-x^{m}\right]$ denotes the irreducible factor of $1-x^{m}$, i.e. the factor which, equated to zero, gives the prime roots of the equation $1-x^{m}=0$. We have

$$
1-x^{m}=\Pi\left[1-x^{m^{\prime}}\right],
$$


where $m^{\prime}$ denotes any divisor whatever of $m$ (unity and the number $m$ itself not excluded). Hence, if a represent a divisor of one or more of the indices $m$, and $k$ be the number of the indices of which $a$ is a divisor, we have

$$
f x=\Pi\left[1-x^{a}\right]^{k} .
$$

Now considering apart from the others one of the multiple factors $\left[1-x^{a}\right]^{k}$, we may write $f x=\left[1-x^{a}\right]^{k} f, x$.

Suppose that the fraction $\frac{\phi x}{f x}$ is decomposed into simpler fractions, in the form

$$
\begin{aligned}
\frac{\phi x}{f^{\prime} x}= & I(x) \\
& +\left(x \partial_{x}\right)^{k-1} \frac{\theta x}{\left[1-x^{a}\right]}+\left(x \partial_{x}\right)^{k-2} \frac{\theta_{1} x}{\left[1-x^{a}\right]} \cdots+\frac{\theta_{k-1} x}{\left[1-x^{a}\right]} \\
& +\& c .,
\end{aligned}
$$

where $I(x)$ denotes the integral part, and the \&c. refers to the fractional terms depending upon the other multiple factors such as $\left[1-x^{a}\right]^{k}$. The functions $\theta x$ are to be considered as functions with indeterminate coefficients, the degree of each such function being inferior by unity to that of the corresponding denominator; and it is proper to remark that the number of the indeterminate coefficients in all the functions $\theta x$ together is equal to the degree of the denominator $f x$.

The term $\left(x \partial_{x}\right)^{k-1} \frac{\theta x}{\left[1-x^{a}\right]}$ may be reduced to the form

$$
\frac{g x}{\left[1-x^{a}\right]^{k}}+\frac{g_{1} x}{\left[1-x^{a}\right]^{k-1}}+\& c .
$$

the functions $g x$ being of the same degree as $\theta x$, and the coefficients of these functions being linearly connected with those of the function $\theta x$. The first of the foregoing terms is the only term on the right-hand side which contains the denominator $\left[1-x^{a}\right]^{k}$; hence, multiplying by this denominator and then writing $\left[1-x^{a}\right]=0$, we find

$$
\frac{\phi x}{f_{1} x}=g x,
$$

which is true when $x$ is any root whatever of the equation $\left[1-x^{a}\right]=0$. Now by means of the equation $\left[1-x^{a}\right]=0, \frac{\phi x}{f_{1} x}$ may be expressed in the form of a rational and integral function $G x$, the degree of which is less by unity than that of $\left[1-x^{a}\right]$. We have therefore $G x=g x$, an equation which is satisfied by each root of $\left[1-x^{a}\right]=0$, and which is therefore an identical equation; $g x$ is thus determined, and the coefficients of $\theta x$ being linear functions of those of $g x$, the function $\theta x$ may be considered as determined. And this being so, the function

$$
\frac{\phi x}{f x}-\left(x \partial_{x}\right)^{k-1} \frac{\theta x}{\left[1-x^{a}\right]}
$$


will be a fraction the denominator of which does not contain any power of $\left[1-x^{a}\right]$ higher than $\left[1-x^{a}\right]^{k-1}$; and therefore $\theta_{1} x$ can be found in the same way as $\theta x$, and similarly $\theta_{2} x$, and so on. And the fractional parts being determined, the integral part may be found by subtracting from $\frac{\phi x}{f x}$ the sum of the fractional parts, so that the fraction $\frac{\phi x}{f x}$ can by a direct process be decomposed in the above-mentioned form.

Particular terms in the decomposition of certain fractions may be obtained with great facility. Thus $m$ being a prime number, assume

$$
\frac{1}{\left(1-x^{2}\right)\left(1-x^{3}\right) \ldots\left(1-x^{m}\right)}=\& c .+\frac{\theta x}{\left[1-x^{m}\right]} ;
$$

then observing that $\left(1-x^{m}\right)=(1-x)\left[1-x^{m}\right]$, we have for $\left[1-x^{m}\right]=0$,

$$
\theta x=\frac{1}{(1-x)\left(1-x^{2}\right) \ldots\left(1-x^{m-1}\right)} .
$$

Now $u$ being any quantity whatever and $x$ being a root of $\left[1-x^{m}\right]=0$, we have identically

$$
\left[1-u^{m}\right]=(u-x)\left(u-x^{2}\right) \ldots\left(u-x^{m-1}\right) ;
$$

and therefore putting $u=1$, we have $m=(1-x)\left(1-x^{2}\right) \ldots\left(1-x^{m-1}\right)$, and therefore

whence

$$
\theta x=\frac{1}{m}
$$

$$
\frac{1}{\left(1-x^{2}\right)\left(1-x^{3}\right) \ldots\left(1-x^{m}\right)}=\& c .+\frac{1}{m} \frac{1}{\left[1-x^{m}\right]} .
$$

Again, $m$ being as before a prime number, assume

$$
\frac{1}{(1-x)\left(1-x^{2}\right) \ldots\left(1-x^{m}\right)}=\& c .+\frac{\theta x}{\left[1-x^{m}\right]},
$$

we have in this case for $\left[1-x^{m}\right]=0$,

$$
\theta x=\frac{1}{(1-x)^{2}\left(1-x^{2}\right) \ldots\left(1-x^{m-1}\right)},
$$

which is immediately reduced to $\theta x=\frac{1}{m} \frac{1}{1-x}$. Now

$\frac{\left[1-u^{m}\right]}{u-x}=\frac{\left[1-u^{m}\right]-\left[1-x^{m}\right]}{u-x}=\left(1+u+\ldots+u^{m-2}\right)+\left(1+u \ldots+u^{m-3}\right) x \ldots+(1+u) x^{m-3}+x^{m-2} ;$

or putting $u=1$,

$$
\frac{m}{1-x}=(m-1)+(m-2) x \ldots+x^{m-2} ;
$$


and substituting this in the value of $\theta x$, we find

$$
\frac{1}{(1-x)\left(1-x^{2}\right) \ldots\left(1-x^{m}\right)}=\& c .+\frac{1}{m^{2}} \frac{(m-1)+(m-2) x \ldots+x^{m-2}}{\left[1-x^{m}\right]} \text {. }
$$

The preceding decomposition of the fraction $\frac{\phi x}{f x}$ gives very readily the expansion of the fraction in ascending powers of $x$. For, consider a fraction such as

$$
\frac{\theta x}{\left[1-x^{a}\right]}
$$

where the degree of the numerator is in general less by unity than that of the denominator; we have

$$
1-x^{a}=\left[1-x^{a}\right] \Pi\left[1-x^{a^{\prime}}\right],
$$

where $a^{\prime}$ denotes any divisor of $a$ (including unity, but not including the number $a$ itself). The fraction may therefore be written under the form

$$
\frac{\theta x \Pi\left[1-x^{a^{\prime}}\right]}{1-x^{a}},
$$

where the degree of the numerator is in general less by unity than that of the denominator, i.e. is equal to $a-1$. Suppose that $b$ is any divisor of $a$ (including unity, but not including the number $a$ itself), then $1-x^{b}$ is a divisor of $\Pi\left[1-x^{a^{\prime}}\right]$, and therefore of the numerator of the fraction. Hence representing this numerator by

$$
A_{0}+A_{1} x \ldots+A_{a-1} x^{a-1},
$$

and putting $a=b c$, we have (corresponding to the case $b=1$ )

$$
A_{0}+A_{1}+A_{2} \ldots+A_{a-1}=0
$$

and generally for the divisor $b$,

$$
\begin{gathered}
A_{0}+A_{b} \ldots+A_{(c-1) b}=0 \\
A_{1}+A_{b+1} \ldots+A_{(c-1) b+1}=0 \\
\vdots \\
A_{b-1}+A_{2 b-1} \ldots+A_{c b-1}=0
\end{gathered}
$$

Suppose now that $a_{q}$ denotes a circulating element to the period $a$, i.e. write

$$
\begin{aligned}
& a_{q}=1, \quad q=0(\bmod . a), \\
& a_{q}=0 \text { in every other case; }
\end{aligned}
$$

a function such as

$$
A_{0} a_{q}+A_{1} a_{q-1} \ldots+A_{a-1} a_{q-a+1}
$$

will be a circulating function, or circulator to the period $a$, and may be represented by the notation

$$
\left(A_{0}, A_{1}, \ldots A_{a-1}\right) \text { circlor } a_{q} \text {. }
$$


In the case however where the coefficients $A$ satisfy, for each divisor $b$ of the number $a$, the above-mentioned equations, the circulating function is what I call a prime circulator, and I represent it by the notation

$$
\left(A_{0}, A_{1}, \ldots A_{a-1}\right) \text { per } a_{q} .
$$

By means of this notation we have at once

$$
\text { coefficient } x^{q} \text { in } \frac{\theta x}{\left[1-x^{a}\right]}=\left(A_{0}, A_{1} \ldots A_{a-1}\right) \text { per } a_{q},
$$

and thence also

$$
\text { coefficient } x^{q} \text { in }\left(x \partial_{x}\right)^{r} \frac{\theta x}{\left[1-x^{a}\right]}=q^{r}\left(A_{0}, A_{1} \ldots A_{a-1}\right) \text { per } a_{q} .
$$

Hence assuming that in the fraction $\frac{\phi x}{f x}$ the degree of the numerator is less than that of the denominator (so that there is not any integral part), we have

$$
\text { coefficient } x^{q} \text { in } \frac{\phi x}{f x}=\Sigma q^{r}\left(A_{0}, A_{1}, \ldots A_{a-1}\right) \text { pcr } a_{q}
$$

or, if we wish to put in evidence the non-circulating part arising from the divisor $a=1$,

$$
\text { coefficient } \begin{aligned}
x^{q} \text { in } \frac{\phi x}{f x}=A q^{k-1}+ & B q^{k-2} \ldots+L q+M \\
& +\Sigma q^{r}\left(A_{0}, A_{1} \ldots A_{a-1}\right) \text { per } a_{q} ;
\end{aligned}
$$

where $k$ denotes the number of the factors $1-x^{m}$ in the denominator $f x, a$ is any divisor (unity excluded) of one or more of the indices $m$; and for each value of $a$ $r$ extends from $r=0$ to $r=k-1$, where $k$ denotes the number of indices $m$ of which $a$ is a divisor. The particular results previously obtained show, that $m$ being a prime number,

and

$$
\text { coefficient } x^{q} \text { in } \frac{1}{\left(1-x^{2}\right)\left(1-x^{3}\right) \ldots\left(1-x^{m}\right)}=\& c .+\frac{1}{m}(\quad 1,-1,0,0, \ldots) \text { pcr } m_{q} \text {, }
$$

$$
\text { coefficient } x^{q} \text { in } \frac{1}{(1-x)\left(1-x^{2}\right) \ldots\left(1-x^{m}\right)}=\& c .+\frac{1}{m^{2}}(m-1,-1,-1, \quad \ldots) \text { per } m_{q} \text {. }
$$

Suppose, as before, that the degree of $\phi x$ is less than that of $f x$, and let the analytical expression above obtained for the coefficient of $x^{q}$ in the expansion in ascending powers of $x$ of the fraction $\frac{\phi x}{f x}$ be represented by $F q$, it is very remarkable that if we expand $\frac{\phi x}{f x}$ in descending powers of $x$, then the coefficient of $x^{q}$ in this new expansion ( $q$ is here of course negative, since the expansion contains only negative powers of $x$ ) is precisely equal to $-F q$; this is in fact at once seen to be 
the case with respect to each of the partial fractions into which $\frac{\phi x}{f x}$ has been decomposed, and it is consequently the case with respect to the fraction itself ${ }^{1}$. This gives rise to a result of some importance. Suppose that $\phi x$ and $f x$ are respectively of the degrees $N$ and $D$; it is clear from the form of $f x$ that we have $f\left(\frac{1}{x}\right)=(-)^{D} x^{-D} f x$; and I suppose that $\phi x$ is also such that $\phi\left(\frac{1}{x}\right)=( \pm)^{N} x^{-N} \phi x$; then writing $D-N=h$, and supposing that $\frac{\phi x}{f x}$ is expanded in descending powers of $x$, so that the coefficient of $x^{q}$ in the expansion is $-F q$, it is in the first place clear that the expansion will commence with the term $x^{-h}$, and we must therefore have

$$
F q=0
$$

for all values of $q$ from $q=-1$ to $q=-(h-1)$.

Consider next the coefficient of a term $x^{-h-q}$, where $q$ is 0 or positive; the coefficient in question, the value of which is $-F(-h-q)$, is obviously equal to the coefficient of $x^{h+q}$ in the expansion in ascending powers of $x$ of $\frac{\phi\left(\frac{1}{x}\right)}{f\left(\frac{1}{x}\right)}$, i.e. to

$$
( \pm)^{N}(-)^{D} \text { coefficient } x^{h+q} \text { in } \frac{x^{h} \phi x}{f x},
$$

or what is the same thing, to

$$
( \pm)^{N}(-)^{D} \text { coefficient } x^{q} \text { in } \frac{\phi x}{f x}
$$

and we have therefore, $q$ being zero or positive,

$$
F(-h-q)=-( \pm)^{N}(-)^{D} F q
$$

In particular, when $\phi x=1$,

$$
F q=0
$$

for all values of $q$ from $q=-1$ to $q=-(D-1)$; and $q$ being 0 or positive,

$$
F(-D-q)=(-)^{D-1} F q \text {. }
$$

The preceding investigations show the general form of the function $P(a, b, c, \ldots) q$, viz. that

$$
P(a, b, c, \ldots) q=A q^{k-1}+B q^{k-2} \ldots+L q+M+\Sigma q^{r}\left(A_{0}, A_{1}, \ldots A_{l-1}\right) \text { per } l_{q},
$$

a formula in which $k$ denotes the number of the elements $a, b, c, \ldots \& c$., and $l$ is any divisor (unity excluded) of one or more of these elements; the summation in the case of each such divisor extends from $r=0$ to $r=k-1$, where $k$ is the number of the elements $a, b, c, \ldots \&$ c. of which $l$ is a divisor; and the investigations indicate

1 The property is a fundamental one in the general theory of developments. 
how the values of the coefficients $A$ of the prime circulators are to be obtained. It has been moreover in effect shown, that if $D=a+b+c+\ldots$, then, writing for shortness $P(q)$ instead of $P(a, b, c, \ldots) q$, we have

$$
P(q)=0
$$

for all values of $q$ from $q=-1$ to $q=-(D-1)$, and that $q$ being 0 or positive,

$$
P(-D-q)=(-)^{D-1} P(q) ;
$$

these last theorems are however uninterpretable in the theory of partitions, and they apply only to the analytical expression for $P(q)$.

I have calculated the following particular results:-

$$
\begin{aligned}
& P(1,2) q=\frac{1}{4}\{2 q+3 \\
& \left.+(1,-1) \text { per } 2_{q}\right\} \\
& P(1,2,3) q \quad=\frac{1}{72}\left\{6 q^{2}+36 q+47\right. \\
& +9(1,-1) \text { per } 2_{q} \\
& \left.+8(2,-1,-1) \text { per } 3_{q}\right\} \\
& P(1,2,3,4) q=\frac{1}{288}\left\{2 q^{3}+30 q^{2}+135 q+175\right. \\
& \left.\begin{array}{l}
+(9 q+45)(1,-1) \text { per } 2_{q} \\
+32 \quad(1,0,-1) \text { pcr } 3_{q} \\
+36 \quad(1,0,-1,0) \text { per } 4_{q}
\end{array}\right\} \\
& P(1,2,3,4,5) q=\frac{1}{86400}\left\{30 q^{4}+900 q^{3}+9300 q^{2}+38250 q+50651\right.
\end{aligned}
$$$$
\left.\begin{array}{l}
+(1350 q+10125) \quad(1,-1) \text { pcr } 2_{q} \\
+3200 \quad(2,-1,-1) \text { per } 3_{q} \\
+5400 \quad(1,1,-1,-1) \text { pcr } 4_{q} \\
+3456(4,-1,-1,-1,-1) \text { per } 5_{q}
\end{array}\right\}
$$

$P(2) q \quad=\frac{1}{2}\{1$

$$
+(1,-1) \text { per } 2 q\}
$$

$$
\begin{aligned}
P(2,3) q \quad=\frac{1}{12}\left\{\begin{array}{l}
2 q \\
+
\end{array}\right. \\
+3 \quad(1,-1) \text { pcr } 2_{q} \\
\left.+4(1,-1,0) \text { per } 3_{q}\right\}
\end{aligned}
$$

C. II. 
where the general term is

$$
x^{\frac{1}{2} s(8+1)} \frac{\left(1-x^{k}\right)\left(1-x^{k-1}\right) \ldots\left(1-x^{k-8+1}\right)}{(1-x)\left(1-x^{2}\right) \ldots\left(1-x^{s}\right)} z^{s},
$$

and the series is a finite one, the last term being that corresponding to $s=k$, viz. $x^{\frac{1 k}{2}(k+1)} z^{k}$. Writing $-x^{m}$ for $z$, and substituting the resulting value of

$$
\left(1-x^{m+1}\right)\left(1-x^{m+2}\right) \ldots\left(1-x^{m+k}\right)
$$

in the formula for $P(0,1,2, \ldots k)^{m} q$, we have

$P(0,1,2, \ldots k)^{m} q=\Sigma_{s}\left\{(-)^{s}\right.$ coefficient $x^{q}$ in $\left.\frac{x^{s m+18(8+1)}}{(1-x)\left(1-x^{2}\right) \ldots\left(1-x^{s}\right)(1-x)\left(1-x^{2}\right) \ldots\left(1-x^{k+8}\right)}\right\}$

where the summation extends from $s=0$ to $s=k$; but if for any value of $s$ between these limits $s m+\frac{1}{2} s(s+1)$ becomes greater than $q$, then it is clear that the summation need only be extended from $s=0$ to the last preceding value of $s$, or what is the same thing, from $s=0$ to the greatest value of $s$ for which $q-s m-\frac{1}{2} s(s+1)$ is positive or zero.

It is obvious, that if $q>k m$, then

$$
P(0,1,2 \ldots k)^{m} q=0 ;
$$

and moreover, that if $\theta \ngtr \frac{1}{2} \mathrm{~km}$, then

$$
P(0,1,2, \ldots k)^{m} \theta=P(0,1,2, \ldots k)^{m} . k m-\theta,
$$

so that we may always suppose $q \ngtr \frac{1}{2} \mathrm{~km}$. I write therefore $q=\frac{1}{2}(k m-\alpha)$ where $\alpha$ is zero or a positive integer not greater than $\mathrm{km}$, and is even or odd according as $k \mathrm{~m}$ is even or odd. Substituting this value of $q$ and making a slight change in the form of the result, we have

$P(0,1,2 \ldots k)^{m} \frac{1}{2}(k m-\alpha)=$

$$
\Sigma_{s}\left\{(-)^{s} \text { coeff. } x^{\left(\frac{1}{2} k-s\right) m} \text { in } \frac{x^{\frac{3}{3 a+1 / s(8+1)}}}{(1-x)\left(1-x^{2}\right) \ldots\left(1-x^{s}\right)(1-x)\left(1-x^{2}\right)\left(1-x^{k-s}\right)}\right\}
$$

where the summation extends from $s=0$ to the greatest value of $s$ for which $\left(\frac{1}{2} k-s\right) m-\frac{1}{2} \alpha-\frac{1}{2} s(s+1)$ is positive or zero. But we may, if we please, consider the summation as extending, when $k$ is even, from $s=0$ to $s=\frac{1}{2} k-1$, and when $k$ is odd from $s=0$ to $s=\frac{1}{2}(k-1)$; the terms corresponding to values of $s$ greater than the greatest value for which $\left(\frac{1}{2} k-s\right) m-\frac{1}{2} \alpha-\frac{1}{2} s(s+1)$ is positive or zero being of course equal to zero. It may be noticed, that the fraction will be a proper one if $\alpha<(k-s)(k-s+1)$; or substituting for $s$ its greatest value, the fraction will be a proper one for all values of $s$, if, when $k$ is even, $\alpha<\frac{1}{4} k(k+2)$, and when $k$ is odd, $\alpha<\frac{1}{4}(k+1)(k+3)$.

We have in a similar manner,

$$
P^{\prime}(0,1,2 \ldots k)^{m} q=\text { coefficient } x^{q} z^{m} \text { in } \frac{1-x}{(1-z)(1-x z) \ldots\left(1-x^{k} z\right)},
$$


which leads to

$P^{\prime}(0,1,2 \ldots k)^{m} \frac{1}{2}(k m-\alpha)=$

$$
\Sigma_{s}\left\{(-)^{s} \text { coeff. } x^{\left(\frac{1}{2} k-s\right) m} \text { in } \frac{x^{\frac{1}{2} a+s(s+1)}}{\left(1-x^{2}\right) \ldots\left(1-x^{s}\right)(1-x)\left(1-x^{2}\right) \ldots\left(1-x^{k-8}\right)}\right\} \text {, }
$$

where the summation extends, as in the former case, from $s=0$ to the greatest value of $s$, for which $\left(\frac{1}{2} k-s\right) m-\frac{1}{2} \alpha-\frac{1}{2} s(s+1)$ is positive or zero, or, if we please, when $k$ is even, from $s=0$ to $s=\frac{1}{2} k-1$, and when $s$ is odd, from $s=0$ to $s=\frac{1}{2}(k-1)$. The condition, in order that the fraction may be a proper one for all values of $s$, is, when $k$ is even, $\alpha+1<\frac{1}{4} k(k+2)$, and when $k$ is odd, $\alpha+1<\frac{1}{4}(k+1)(k+3)$.

To transform the preceding expressions, I write when $k$ is odd $x^{2}$ instead of $x$, and I put for shortness $\theta$ instead of $\frac{1}{2} k-s$ or $2\left(\frac{1}{2} k-s\right)$, and $\gamma$ instead of $\frac{1}{2} \alpha+\frac{1}{2} s(s+1)$ or $\alpha+s(s+1)$; we have to consider an expression of the form

$$
\text { coefficient } x^{\theta m} \text { in } \frac{x^{\gamma}}{F x}
$$

where $F x$ is the product of factors of the form $1-x^{a}$. Suppose that $a^{\prime}$ is the least common multiple of $a$ and $\theta$, then $\left(1-x^{a^{\prime}}\right) \div\left(1-x^{a}\right)$ is an integral function of $x$, equal $\chi^{x}$ suppose, and $1 \div\left(1-x^{a}\right)=\chi x \div\left(1-x^{a^{\prime}}\right)$. Making this change in all the factors of $F x$ which require it (i.e. in all the factors except those in which $a$ is a multiple of $\theta$ ), the general term becomes

$$
\text { coefficient } x^{\theta m} \text { in } \frac{x^{\gamma} H x}{G x},
$$

where $G x$ is a product of factors of the form $1-x^{a^{\prime}}$, in which $a^{\prime}$ is a multiple of $\theta$, i.e. $G x$ is a rational and integral function of $x^{\theta}$. But in the numerator $x^{\gamma} H x$ we may reject, as not contributing to the formation of the coefficient of $x^{\theta m}$, all the terms in which the indices are not multiples of $\theta$; the numerator is thus reduced to a rational and integral function of $x^{\theta}$, and the general term is therefore of the form

$$
\text { coefficient } x^{\theta m} \text { in } \frac{\lambda\left(x^{\theta}\right)}{\kappa\left(x^{\theta}\right)},
$$

or what is the same thing, of the form

$$
\text { coefficient } x^{m} \text { in } \frac{\lambda x}{\kappa x} \text {, }
$$

where $\kappa x$ is the product of factors of the form $1-x^{a}$, and $\lambda x$ is a rational and integral function of $x$. The particular value of the fraction depends on the value of $s$; and uniting the different terms, we have an expression

$$
\text { coefficient } x^{m} \text { in } S_{s}(-)^{s} \frac{\lambda x}{\kappa x}
$$

which is equivalent to

$$
\text { coefficient } x^{m} \text { in } \frac{\phi x}{f x}
$$


where $f x$ is a product of factors of the form $1-x^{a}$, and $\phi x$ is a rational and integral function of $x$. And it is clear that the fraction will be a proper one when each of the fractions in the original expression is a proper fraction, i.e. in the case of $P(0,1,2 \ldots k)^{m} \frac{1}{2}(k m-\alpha)$, when for $k$ even, $\alpha<\frac{1}{4} k(k+2)$, and for $k$ odd, $\alpha<\frac{1}{4}(k+1)(k+3)$; and in the case of $P^{\prime}(0,1,2 \ldots k)^{m} \frac{1}{2}(k m-\alpha)$, when for $k$ even, $\alpha+1<\frac{1}{4} k(k+2)$, and for $k$ odd, $\alpha+1<\frac{1}{4}(k+1)(k+3)$.

We see, therefore, that

$$
P(0,1,2 \ldots k)^{m} \frac{1}{2}(k m-\alpha),
$$

and

$$
P^{\prime}(0,1,2 \ldots ;)^{m} \frac{1}{2}(k m-\alpha),
$$

are each of them of the form

$$
\text { coefficient } x^{m} \text { in } \frac{\phi x}{f x} \text {, }
$$

where $f x$ is the product of factors of the form $1-x^{a}$, and up to certain limiting values of $\alpha$ the fraction is a proper fraction. When the fraction $\frac{\phi x}{f x}$ is known, we may therefore obtain by the method employed in the former part of this Memoir, analytical expressions (involving prime circulators) for the functions $P$ and $P^{\prime}$.

As an example, take

which is equal to

$$
P(0,1,2,3)^{m} \frac{3}{2} m,
$$

$$
\begin{aligned}
& \text { coefficient } x^{3 m} \text { in } \frac{1}{\left(1-x^{2}\right)\left(1-x^{4}\right)\left(1-x^{6}\right)} \\
& - \text { coefficient } x^{m} \text { in } \frac{1}{\left(1-x^{2}\right)\left(1-x^{2}\right)\left(1-x^{4}\right)} .
\end{aligned}
$$

The multiplier for the first fraction is

which is equal to

$$
\frac{\left(1-x^{6}\right)\left(1-x^{12}\right)}{\left(1-x^{2}\right)\left(1-x^{4}\right)}
$$

$$
1+x^{2}+2 x^{4}+x^{6}+2 x^{8}+x^{10}+x^{12} .
$$

Hence, rejecting in the numerator the terms the indices of which are not divisible by 3 , the first term becomes

$$
\text { coefficient } x^{3 m} \text { in } \frac{1+x^{6}+x^{12}}{\left(1-x^{6}\right)\left(1-x^{12}\right)\left(1-x^{6}\right)} \text {, }
$$

or what is the same thing, the first term is

$$
\text { coefficient } x^{m} \text { in } \frac{1+x^{2}+x^{4}}{\left(1-x^{2}\right)^{2}\left(1-x^{4}\right)}
$$


and, the second term being

$$
- \text { coefficient } x^{m} \text { in } \frac{x^{2}}{\left(1-x^{2}\right)^{2}\left(1-x^{4}\right)} ;
$$

we have

$$
P(0,1,2,3)^{m} \frac{3}{2} m=\text { coefficient } x^{m} \text { in } \frac{1+x^{4}}{\left(1-x^{2}\right)^{2}\left(1-x^{4}\right)}
$$

and similarly it may be shown, that

$$
P(0,1,2,3)^{m} \frac{1}{2}(3 m-1)=\text { coefficient } x^{m} \text { in } \frac{x+x^{3}}{\left(1-x^{2}\right)^{2}\left(1-x^{4}\right)} .
$$

As another example, take

which is equal to

$$
P^{\prime}(0,1,2,3,4,5) \frac{5}{2} m
$$

$$
\begin{aligned}
& \text { coefficient } x^{5 m} \text { in } \frac{1}{\left(1-x^{4}\right)\left(1-x^{6}\right)\left(1-x^{8}\right)\left(1-x^{10}\right)} \\
& - \text { coefficient } x^{3 m} \text { in } \frac{x^{2}}{\left(1-x^{2}\right)\left(1-x^{4}\right)\left(1-x^{6}\right)\left(1-x^{8}\right)} \\
& + \text { coefficient } x^{m} \text { in } \frac{x^{6}}{\left(1-x^{2}\right)\left(1-x^{4}\right)\left(1-x^{4}\right)\left(1-x^{6}\right)} .
\end{aligned}
$$

The multiplier for the first fraction is

$$
\frac{\left(1-x^{20}\right)\left(1-x^{30}\right)\left(1-x^{40}\right)}{\left(1-x^{4}\right)\left(1-x^{6}\right)\left(1-x^{8}\right)}
$$

which is a function of $x^{2}$ of the order 36 , the coefficients of which are

$$
1,0,1,1,2,1,3,2,4,3,4,4,6,4,6,5,7,5,7,5,7,5,6,4,6,4,4,3,4,2,3,1,2,1,1,0,1 \text {, }
$$
and the first part becomes therefore

$$
\text { coefficient } x^{m} \text { in } \frac{1+x^{2}+4 x^{4}+5 x^{6}+7 x^{8}+4 x^{10}+3 x^{12}}{\left(1-x^{2}\right)\left(1-x^{4}\right)\left(1-x^{6}\right)\left(1-x^{8}\right)} \text {. }
$$

The multiplier for the second fraction is

$$
\frac{\left(1-x^{6}\right)\left(1-x^{12}\right)\left(1-x^{24}\right)}{\left(1-x^{2}\right)\left(1-x^{4}\right)\left(1-x^{8}\right)}
$$

which is a function of $x^{2}$ of the order 14, the coefficients of which are

$$
1,1,2,1,3,2,3,1,3,2,3,1,2,1,1 \text {; }
$$

and the second term becomes

$$
- \text { coefficient } x^{m} \text { in } \frac{2 x^{2}+2 x^{4}+3 x^{6}+x^{8}+x^{10}}{\left(1-x^{2}\right)^{2}\left(1-x^{4}\right)\left(1-x^{8}\right)}
$$


and the third term is

$$
\text { coefficient } x^{m} \text { in } \frac{x^{6}}{\left(1-x^{2}\right)\left(1-x^{4}\right)^{2}\left(1-x^{6}\right)} \text {. }
$$

Now the fractions may be reduced to a common denominator

$$
\left(1-x^{2}\right)\left(1-x^{4}\right)\left(1-x^{6}\right)\left(1-x^{8}\right)
$$

by multiplying the terms of the second fraction by $\frac{1-x^{6}}{1-x^{2}}\left(=1+x^{2}+x^{4}\right)$, and the terms of the third fraction by $\frac{1-x^{8}}{1-x^{4}}\left(=1+x^{4}\right)$; performing the operations and adding, the numerator and denominator of the resulting fraction will each of them contain the factor $1-x^{2}$; and casting this out, we find

$$
P(0,1,2,3,4,5)^{m} \frac{5}{2} m=\text { coefficient } x^{m} \text { in } \frac{1-x^{6}+x^{12}}{\left(1-x^{4}\right)\left(1-x^{6}\right)\left(1-x^{8}\right)} .
$$

I have calculated by this method several other particular cases, which are given in my "Second Memoir upon Quantics", [141], the present researches were in fact made for the sake of their application to that theory.

Received April 20,-_Read May 3 and 10, 1855.

Since the preceding portions of the present Memoir were written, Mr Sylvester has communicated to me a remarkable theorem which has led me to the following additional investigations ${ }^{1}$.

Let $\frac{\phi x}{f x}$ be a rational fraction, and let $\left(x-x_{1}\right)^{k}$ be a factor of the denominator $f x$, then if

$$
\left\{\frac{\phi x}{f x}\right\}_{x_{1}}
$$

denote the portion which is made up of the simple fractions having powers of $x-x_{1}$ for their denominators, we have by a known theorem

$$
\left\{\frac{\phi x}{f x}\right\}_{x_{1}}=\text { coefficient } \frac{1}{z} \text { in } \frac{1}{x-x_{1}-z} \frac{\phi\left(x_{1}+z\right)}{f\left(x_{1}+z\right)} .
$$

Now by a theorem of Jacobi's and Cauchy's,

$$
\text { coefficient } \frac{1}{z} \text { in } F z=\text { coefficient } \frac{1}{t} \text { in } F(\psi t) \psi^{\prime} t \text {; }
$$

whence, writing $x_{1}+z=x_{1} e^{-t}$, we have

$$
\left\{\frac{\phi x}{f x}\right\}_{x_{1}}=\text { coefficient } \frac{1}{t} \text { in } \frac{x_{1}}{x_{1}-x e^{t}} \frac{\phi\left(x_{1} e^{-t}\right)}{f\left(x_{1} e^{-t}\right)} .
$$

$1 \mathrm{Mr}$ Sylvester's researches are published in the Quarterly Mathematical Journal, July 1855, [vol. 1. pp. 141-152], and he has there given the general formula as well for the eirculating as the non-circulating part of the expression for the number of partitions.-Added 23rd February, 1856.-A. C. 
Now putting for a moment $x=x_{1} e^{\theta}$, we have

$$
\frac{1}{x_{1}-x e^{t}}=\frac{1}{x_{1}\left(1-e^{\theta+t}\right)}=\frac{1}{x_{1}\left(1-e^{\theta}\right)}+\partial_{\theta} \frac{1}{x_{1}\left(1-e^{\theta}\right)}+\ldots
$$

and $\partial_{\theta}=x \partial_{x}$, whence

$$
\frac{1}{x_{1}-x e^{t}}=\frac{1}{x_{1}-x}+\frac{t}{1} x \partial_{x} \frac{1}{x_{1}-x}+\frac{t^{2}}{1.2}\left(x \partial_{x}\right)^{2} \frac{1}{x_{1}-x}+\ldots
$$

the general term of which is

$$
\frac{t^{s-1}}{\Pi(s-1)}\left(x \partial_{x}\right)^{s-1} \frac{1}{x_{1}-x} .
$$

Hence representing the general term of

by $\chi^{x_{1}} t^{-s}$, so that

$$
\frac{x_{1} \phi\left(x_{1} e^{-t}\right)}{f\left(x_{1} e^{-t}\right)}
$$

$$
\chi^{x_{1}}=\text { coefficient } \frac{1}{t} \text { in } t^{s-1} \frac{x_{1} \phi\left(x_{1} e^{-t}\right)}{f\left(x_{1} e^{-t}\right)}
$$

we find, writing down only the general term,

$$
\left\{\frac{\phi x}{f x}\right\}_{x_{1}}=\ldots+\frac{1}{\Pi(s-1)}\left(x \partial_{x}\right)^{s-1} \frac{\chi^{x_{1}}}{x_{1}-x}+\ldots,
$$

where the value of $\chi^{x_{1}}$ depends upon that of $s$, and where $s$ extends from $s=1$ to $s=k$.

Suppose now that the denominator is made up of factors (the same or different) of the form $1-x^{m}$. And let $a$ be any divisor of one or more of the indices $m$, and let $k$ be the number of the indices of which $a$ is a divisor. The denominator contains the divisor $\left[1-x^{a}\right]^{k}$, and consequently if $\rho$ be any root of the equation $\left[1-x^{a}\right]=0$, the denominator contains the factor $(\rho-x)^{k}$. Hence writing $\rho$ for $x_{1}$ and taking the sum with respect to all the roots of the equation $\left[1-x^{a}\right]=0$, we find

$$
\begin{aligned}
\left\{\frac{\phi x}{f x}\right\}_{\left[1-x^{a}\right]} & =\ldots+\frac{1}{\Pi(s-1)}\left(x \partial_{x}\right)^{s-1} S \frac{\chi \rho}{\rho-x}+\ldots \\
& =\ldots+\frac{1}{\Pi(s-1)}\left(x \partial_{x}\right)^{s-1} \frac{\theta x}{\left[1-x^{a}\right]}+\ldots
\end{aligned}
$$

where

$$
\chi \rho=\text { coefficient } \frac{1}{t} \text { in } t^{s-1} \frac{\rho \phi\left(\rho e^{-t}\right)}{f\left(\rho e^{-t}\right)},
$$

and as before $s$ extends from $s=1$ to $s=k$. We have thus the actual value of the function $\theta x$ made use of in the memoir.

A preceding formula gives

$$
\left\{\frac{\phi x}{f x}\right\}_{1}=\text { coefficient } \frac{1}{t} \text { in } \frac{1}{1-x e^{t}} \frac{\phi\left(e^{-t}\right)}{f\left(e^{-t}\right)}
$$

which is a very simple expression for the non-circulating part of the fraction $\frac{\phi x}{f x}$. This is, in fact, Mr Sylvester's theorem above referred to.

C. II. 
and the third term is

$$
\text { coefficient } x^{m} \text { in } \frac{x^{6}}{\left(1-x^{2}\right)\left(1-x^{4}\right)^{2}\left(1-x^{6}\right)} \text {. }
$$

Now the fractions may be reduced to a common denominator

$$
\left(1-x^{2}\right)\left(1-x^{4}\right)\left(1-x^{6}\right)\left(1-x^{8}\right)
$$

by multiplying the terms of the second fraction by $\frac{1-x^{6}}{1-x^{2}}\left(=1+x^{2}+x^{4}\right)$, and the terms of the third fraction by $\frac{1-x^{8}}{1-x^{4}}\left(=1+x^{4}\right)$; performing the operations and adding, the numerator and denominator of the resulting fraction will each of them contain the factor $1-x^{2}$; and casting this out, we find

$$
P(0,1,2,3,4,5)^{m} \frac{5}{2} m=\text { coefficient } x^{m} \text { in } \frac{1-x^{6}+x^{12}}{\left(1-x^{4}\right)\left(1-x^{6}\right)\left(1-x^{8}\right)} .
$$

I have calculated by this method several other particular cases, which are given in my "Second Memoir upon Quantics", [141], the present researches were in fact made for the sake of their application to that theory.

\section{Received April 20,-Read May 3 and 10, 1855.}

Since the preceding portions of the present Memoir were written, $\mathrm{Mr}$ Sylvester has communicated to me a remarkable theorem which has led me to the following additional investigations ${ }^{1}$.

Let $\frac{\phi x}{f x}$ be a rational fraction, and let $\left(x-x_{1}\right)^{k}$ be a factor of the denominator $f x$, then if

$$
\left\{\frac{\phi x}{f x}\right\}_{x_{1}}
$$

denote the portion which is made up of the simple fractions having powers of $x-x_{1}$ for their denominators, we have by a known theorem

$$
\left\{\frac{\phi x}{f x}\right\}_{x_{1}}=\text { coefficient } \frac{1}{z} \text { in } \frac{1}{x-x_{1}-z} \frac{\phi\left(x_{1}+z\right)}{f\left(x_{1}+z\right)} .
$$

Now by a theorem of Jacobi's and Cauchy's,

$$
\text { coefficient } \frac{1}{z} \text { in } F z=\text { coefficient } \frac{1}{t} \text { in } F(\psi t) \psi^{\prime} t \text {; }
$$

whence, writing $x_{1}+z=x_{1} e^{-t}$, we have

$$
\left\{\frac{\phi x}{f x}\right\}_{x_{1}}=\text { coefficient } \frac{1}{t} \text { in } \frac{x_{1}}{x_{1}-x e^{t}} \frac{\phi\left(x_{1} e^{-t}\right)}{f\left(x_{1} e^{-t}\right)} .
$$

$1 \mathrm{Mr}$ Sylvester's researches are published in the Quarterly Mathematical Journal, July 1855, [vol, 1. p? 141-152], and he has there given the general formula as well for the circulating as the non-circulating part of the expression for the number of partitions.-Added 23rd February, 1856.-A. C. 
Now putting for a moment $x=x_{1} e^{\theta}$, we have

$$
\frac{1}{x_{1}-x e^{t}}=\frac{1}{x_{1}\left(1-e^{\theta+t}\right)}=\frac{1}{x_{1}\left(1-e^{\theta}\right)}+\partial_{\theta} \frac{1}{x_{1}\left(1-e^{\theta}\right)}+\ldots
$$

and $\partial_{\theta}=x \partial_{x}$, whence

$$
\frac{1}{x_{1}-x e^{t}}=\frac{1}{x_{1}-x}+\frac{t}{1} x \partial_{x} \frac{1}{x_{1}-x}+\frac{t^{2}}{1.2}\left(x \partial_{x}\right)^{2} \frac{1}{x_{1}-x}+\ldots
$$

the general term of which is

$$
\frac{t^{s-1}}{\Pi(s-1)}\left(x \partial_{x}\right)^{s-1} \frac{1}{x_{1}-x} .
$$

Hence representing the general term of

by $\chi x_{1} t^{-8}$, so that

$$
\frac{x_{1} \phi\left(x_{1} e^{-t}\right)}{f\left(x_{1} e^{-t}\right)}
$$

$$
\chi^{x_{1}}=\text { coefficient } \frac{1}{t} \text { in } t^{s-1} \frac{x_{1} \phi\left(x_{1} e^{-t}\right)}{f\left(x_{1} e^{-t}\right)},
$$

we find, writing down only the general term,

$$
\left\{\frac{\phi x}{f x}\right\}_{x_{1}}=\ldots+\frac{1}{\Pi(s-1)}\left(x \partial_{x}\right)^{s-1} \frac{\chi^{x_{1}}}{x_{1}-x}+\ldots,
$$

where the value of $\chi x_{1}$ depends upon that of $s$, and where $s$ extends from $s=1$ to $s=k$.

Suppose now that the denominator is made up of factors (the same or different) of the form $1-x^{m}$. And let $a$ be any divisor of one or more of the indices $m$, and let $k$ be the number of the indices of which $a$ is a divisor. The denominator contains the divisor $\left[1-x^{a}\right]^{k}$, and consequently if $\rho$ be any root of the equation $\left[1-x^{a}\right]=0$, the denominator contains the factor $(\rho-x)^{k}$. Hence writing $\rho$ for $x_{1}$ and taking the sum with respect to all the roots of the equation $\left[1-x^{a}\right]=0$, we find

$$
\begin{aligned}
\left\{\frac{\phi x}{f x}\right\}_{\left[1-x^{a}\right]} & =\ldots+\frac{1}{\Pi(s-1)}\left(x \partial_{x}\right)^{s-1} S \frac{\chi \rho}{\rho-x}+\ldots \\
& =\ldots+\frac{1}{\Pi(s-1)}\left(x \partial_{x}\right)^{s-1} \frac{\theta x}{\left[1-x^{a}\right]}+\ldots
\end{aligned}
$$

where

$$
\chi \rho=\text { coefficient } \frac{1}{t} \text { in } t^{s-1} \frac{\rho \phi\left(\rho e^{-t}\right)}{f\left(\rho e^{-t}\right)},
$$

and as before $s$ extends from $s=1$ to $s=k$. We have thus the actual value of the function $\theta x$ made use of in the memoir.

A preceding formula gives

$$
\left\{\frac{\phi x}{f x}\right\}_{1}=\text { coefficient } \frac{1}{t} \text { in } \frac{1}{1-x e^{t}} \frac{\phi\left(e^{-t}\right)}{f\left(e^{-t}\right)},
$$

which is a very simple expression for the non-circulating part of the fraction $\frac{\phi x}{f x}$.

This is, in fact, Mr Sylvester's theorem above referred to.

C. II. 